\title{
Atomic Manipulation on a Scanning Transmission Electron Microscope Platform using Real-Time Image Processing and Feedback
}

\author{
Ondrej Dyck ${ }^{1,2}$, Songkil Kim ${ }^{2,3}$, Albina Borisevich ${ }^{2,4}$, Bethany M. Hudak ${ }^{2,4}$, Andrew R. Lupini ${ }^{2,4}$, Sergei \\ V. Kalinin ${ }^{1,2}$, and Stephen Jesse ${ }^{1,2}$ \\ 1. Center for Nanophase Materials Sciences, Oak Ridge National Laboratory, Oak Ridge, TN USA \\ 2. Institute for Functional Imaging of Materials, Oak Ridge National Laboratory, Oak Ridge, TN USA \\ 3. Department of Mechanical Engineering, Pusan National University, Republic of Korea \\ 4. Materials Science and Technology Division, Oak Ridge National Laboratory, Oak Ridge, TN USA
}

Fabrication of atomic scale structures remains the ultimate goal of nanotechnology. The reigning paradigms have been scanning probe microscopy (SPM) and synthesis. SPM assembly dates to seminal experiments by Don Eigler, who demonstrated single atom manipulation. However, stability and throughput remain issues. Discuss here are research activity towards the next paradigm - the use of the atomically focused beam of a scanning transmission electron microscope (STEM) to control and direct matter on atomic scales. Traditionally, STEM's are perceived only as imaging tools and beam induced modifications as undesirable beam damage. Our team and several groups worldwide have demonstrated that beam induced modifications can be more precise and controllable. We have demonstrated ordering of oxygen vacancies, single defect formation in 2D materials including adding and moving dopants within a lattice [1-4], and beam induced migration of single interstitials in diamond like materials. What is remarkable is that these changes often involve one atom or small group of atoms and can be monitored real-time with atomic resolution.

Critical to controlled manipulation of matter at the atomic scale is the ability to have an automated system to process multiple streams of data in real-time, operate on the processed data, and make fast decisions based on this information to correctly set optimal conditions (such as beam position and trajectpru) to induce the desired transformation. Further complications arise since one uses the same beam to both image material and change it. Therefore, it is necessary to develop imaging methods that introduce as small of an electron dose as possible to the material during imaging while still providing sufficient reliability as well as develop schemes to modify materials with maximal effectiveness during atomic scale manipulation. Several examples will be introduced of beam-induced fabrication on the atomic level, and demonstration of how beam control, rapid image analytics, better insight through modelling, and image-based feedback allows for controlling matter on the atomic level.

\section{References:}

[1] Dyck O., Kim S., Kalinin S., Jesse S., "Assembling Di-and Multiatomic Si Clusters in Graphene via Electron Beam Manipulation", ARXIV:1710.09416 (2017)

[2] Dyck O., Kim S., Kalinin S., Jesse S., "E-beam manipulation of Si atoms on graphene edges with aberration-corrected STEM", ARXIV:1710.10338 (2017)

[3] Dyck O., Kim S., Kalinin S., Jesse S., "Mitigating e-beam-induced hydrocarbon deposition on graphene for atomic-scale scanning transmission electron microscopy studies", JOURNAL OF VACUUM SCIENCE \& TECHNOLOGY B 36 (2018)

[4] Dyck O., Kim S., Kalinin S. V., Jesse S., "Placing single atoms in graphene with a scanning transmission electron microscope", APPLIED PHYSICS LETTERS 111 (2017) 
[5] Kalinin S. V., Borisevich A., Jesse S., "Fire up the Atom Forge", NATURE 539 (2016)

[6] Jesse S., et. al., "Atomic-Level Sculpting of Crystalline Oxides: Toward Bulk Nanofabrication with Single Atomic Plane Precision", Small 11 (2015)

[7] Research supported by Oak Ridge National Laboratory's Center for Nanophase Materials

Sciences (CNMS), which is sponsored by the Scientific User Facilities Division, Office of Basic

Energy Sciences, U.S. Department of Energy (S.V.K.), and by the Laboratory Directed Research and

Development Program of Oak Ridge National Laboratory, managed by UT-Battelle, LLC, for the U.S.

Department of Energy (O.D, S.K., S.J.).

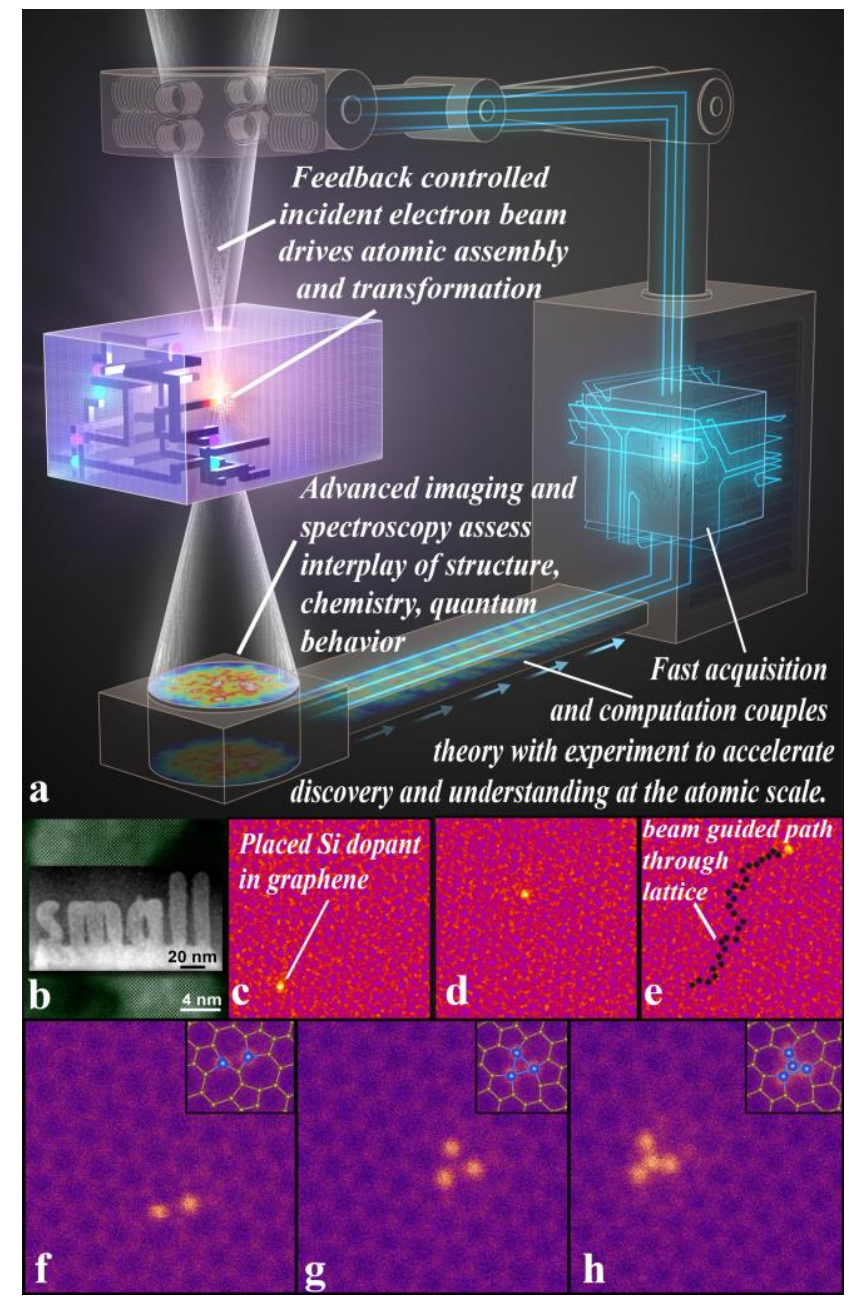

Figure 1. Information Flow for Directed Atomic Assembly in STEM. (a) Enhanced STEMs enable building and editing of atomic assemblies and assessment of their chemical, physical, and quantum properties [5]. (b) Layer-by-layer atomic scale sculpting [6]. (c-e) Directed doping and guided motion of $\mathrm{Si}$ atom through graphene lattice. (f-h) Beam directed atom-by-atom fabrication of Si dimer, trimer, and tetratramer. 